\title{
A Markov Regime-Switching Model with Time-Varying Transition Probabilities for Identifying Asset Price Bubbles
}

\author{
Matthew L. Higgins ${ }^{1} \&$ Frank Ofori-Acheampong ${ }^{1}$ \\ ${ }^{1}$ Department of Economics, Western Michigan University, USA \\ Correspondence: Frank Ofori-Acheampong, Department of Economics, Western Michigan University, $1903 \mathrm{~W}$ \\ Michigan Avenue, Kalamazoo, MI 49008-5330, USA. E-mail: frank.ofori@wmich.edu; fka.ofori@gmail.com
}

Received: January 13, 2018

Accepted: February 18, 2018

Online Published: February 28, 2018

doi:10.5539/ijef.v10n4p1

URL: https://doi.org/10.5539/ijef.v10n4p1

\begin{abstract}
In this paper, a Markov regime-switching model with time-varying transition probabilities is developed to identify asset price bubbles in the S\&P 500 index. The model nests two different methodologies; a state-dependent regime-switching model and a Markov regime-switching model. Three bubble regimes are identified; dormant, explosive, and collapsing. Time-varying transition probabilities are specified for each of the nine possible transitions in the Markov regime-switching model. Estimation of the model is done using conditional maximum likelihood with the Hamilton filter. Results show that transition probabilities depend significantly on trading volume and relative size of the bubble. Overall, the model works well in detecting multiple bubbles in the S\&P 500 between January 1888 and May 2010. Explosive bubbles tend to immediately precede recession periods, while collapsing bubbles tend to coincide with recession periods.
\end{abstract}

Keywords: asset price bubble, Markov regime-switching, recession, S\&P 500 index, time-varying transition probability

\section{Introduction}

Observed movements in the S\&P 500 index can be difficult to explain by fundamental factors. The deviation of asset prices from their fundamental values have often been attributed to the presence of rational bubbles. A rational bubble is a large and persistent deviation of the market price of an asset from its fundamental value resulting from rational speculative behavior. Figure 1 shows the monthly S\&P 500 price index from January 1888 to May 2010 together with NBER recession dates over the same period. Stock market booms and busts are often seemingly linked to the business cycle. This is apparent in the period prior to the Great Depression in which the index shows steady growth up to 1922, then evolves into explosive growth from 1923 to 1929 and later collapses from 1929 to 1933. Similar patterns are observed in latter periods such as the late 1990's and early 2000 's associated with the internet bubble and the Great Recession. In each of these cases, the stock price collapse coincided with a recession. The sequence of dormant, explosive and collapsing bubbles is not always consistent nor is it always associated with the business cycle. For example, periods of dormant bubbles appear to be followed immediately by a collapse in 1900-1902, 1905-1907 and 1978-1981. Similarly, a collapse can be followed by an apparently explosive bubble as in 1962, 1974, 2003 and 2009. Finally, an explosive bubble can be followed by a dormant bubble as in 1990-1994 and 2004-2006.

Two strands of literature, often characterized as the indirect approach and direct approach, exist on empirically detecting the presence of asset price bubbles. The indirect approach was initiated by Diba and Grossman (1987, 1988) and recently revitalized by Phillips, Wu and Yu (2011) and Phillips, Shi and Yu (2015a, 2015b). In this approach, bubbles are detected through the time series properties of prices and dividends, specifically by showing that prices have explosive autoregressive roots and demonstrating that prices and dividends are not cointegrated. In the direct approach, an explicit model is specified for the latent periodically collapsing bubble process and the parameters of the process are estimated by maximum likelihood or Bayesian methods. Within the direct approach, two sub-strands of the literature exist. The first approach follows Evans (1991) in which the bubble regime transition probabilities are governed by state variables such as the size of the bubble and trading volume. This line of research has been developed in a series of papers by van Norden and Schaller $(1993,2002)$, van Norden and Vigfusson (1998), Brooks and Katsaris (2005) and Anderson, Brooks and Katsaris (2010). In the second approach, bubble regime transitions are completely exogenous and are governed by a multiple state 
Markov process. Papers which employ this approach include Fulop and Yu (2017), Shi and Song (2014), and Balke and Wohar (2009).

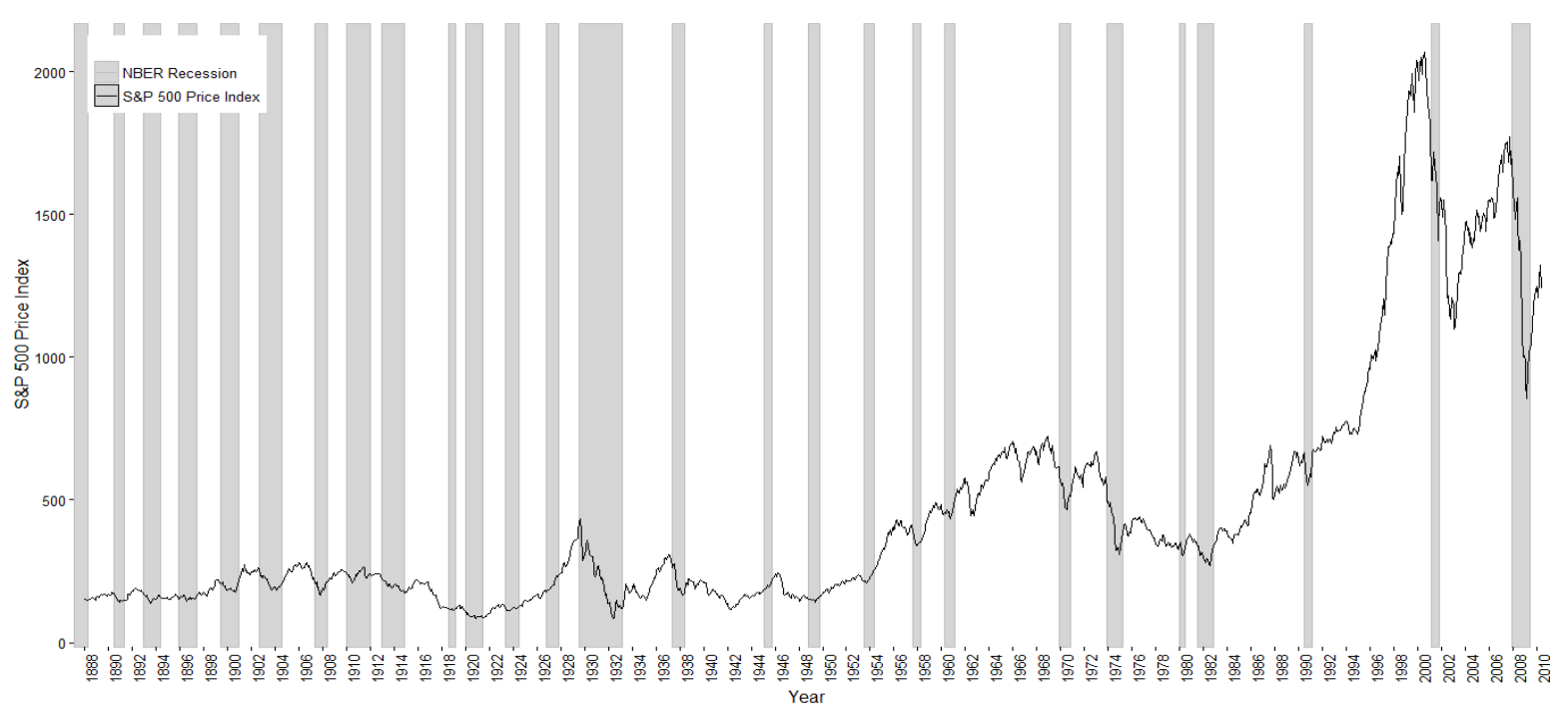

Figure 1. S\&P 500 stock price index and NBER recession periods between January 1888 and May 2010

In this paper, we follow the direct approach and estimate an explicit model for a periodically collapsing bubble in the S\&P 500 index. Our contribution is that we combine the state-dependent approach with the Markov switching approach. We estimate a very general three-regime bubble process with dormant, explosive and collapsing states in which the regimes follow a Markov switching process with state-dependent transition probabilities. By combining the Markov and state-dependent models, the effect of a state variable can depend on the current bubble regime. For example, we find that when the bubble is in the dormant regime, the probability of exiting the regime increases with the relative size of the bubble. In contrast, when the bubble is in the explosive regime, the probability of leaving the explosive regime is independent of all the state variables. Moreover, when the bubble is in the collapsing regime, the probability of reverting to an explosive regime increases with the relative bubble size. These complex probabilities can explain why we do not always observe a sequential transition from dormant to explosive to collapsing regimes of bubbles in the S\&P 500 index. Results show that the three-regime Markov switching speculative bubble model is able to identify most of the historical bubble phenomena, such as 'The Great Depression,' 'Black Monday,' 'Friday the 13th,' the Kennedy slide (flash crash) and the 'Internet Bubble.'

\section{A Three-Regime Markov Switching Rational Speculative Bubble Model}

In this section, the three-regime model by Brooks and Katsaris (hereafter called BK model) is adapted to a Markov regime-switching model. To model the bubble behavior, we begin with the present value model

$$
P_{t}=(1+R)^{-1} E_{t}\left(P_{t+1}+D_{t+1}\right)
$$

where $P_{t}$ is the asset price at time $t, R$ is a constant rate of discount, $D_{t+1}$ is the dividend payment at time $t+1$. By imposing the transversality condition, a particular solution to (1) is

$$
P_{t}^{f} \equiv \sum_{k=1}^{\infty}(1+R)^{-k} E_{t} D_{t+k}
$$

where $P_{t}^{f}$ denotes the fundamental price of an asset. The solution to (1) may contain another component besides $P_{t}^{f}$. Let $B_{t}=P_{t}-P_{t}^{f}$. If

$$
B_{t}=(1+R)^{-1} E_{t} B_{t+1}
$$

then $P_{t}=P_{t}^{f}+B_{t}$ is also a solution to (1). The component $B_{t}$ is called a bubble.

Having identified the form of the bubble, the three-regime model for the bubble process in period $t+1$ is then formulated. Define an indicator variable $S_{t}$ such that

$$
S_{t}= \begin{cases}1, & \text { if } B_{t} \text { is dormant } \\ 2, & \text { if } B_{t} \text { is explosive } \\ 3, & \text { if } B_{t} \text { is collapsing }\end{cases}
$$


If the dormant bubble regime occurs in period $t+1$, the bubble will grow at a constant mean rate of $(1+R)$ such that

$$
E_{t}\left(B_{t+1} \mid S_{t+1}=1\right)=(1+R) B_{t} .
$$

In this regime, the bubble does not collapse and thus investors have no expectations of large deviations in asset returns. Denote the probability of being in the dormant regime by $n_{t}$ which is state-dependent, and will also depend on the relative size of the bubble as well as the spread between fundamental returns and actual returns on the S\&P 500 index. Later, we add a Markov chain structure in which the regime probabilities also depend upon the previous regime. We omit this for now to simplify notation. The probability of being in the non-dormant regime is given by $1-n_{t}$. In the non-dormant state, two underlying regimes are defined: the explosive regime that occurs with probability $q_{t}$ and the collapsing regime that occurs with probability $1-q_{t}$. We specify $q_{t}$ as a function of the relative bubble size and volume traded of the $S \& P 500$ index. If the collapsing regime occurs

$$
E_{t}\left(B_{t+1} \mid S_{t+1}=3\right)=g\left(b_{t}\right) P_{t} /\left(1-q_{t}\right),
$$

where $g\left(b_{t}\right)$ is a continuous and everywhere differentiable function with $g\left(b_{t}\right)>0$ and $0<\partial g\left(b_{t}\right) / \partial b_{t}<1+$ $R$. $b_{t}$ is the relative bubble size defined by $b_{t}=B_{t} / P_{t}$. The restriction on $\partial g\left(b_{t}\right) / \partial b_{t}$ ensures that the bubble in the collapsing regime grows slower than that under the dormant regime. One distinction between equation (5) and that specified in the BK model is the multiplication by $1 /\left(1-q_{t}\right)$. As the probability of being in the explosive regime $q_{t}$ increases, the collapsing regime is less likely to occur with probability $1-q_{t}$. However, if the collapsing regime does occur, then the collapse from a higher price level will be more severe that the collapse from a lower price level. Thus, $1 /\left(1-q_{t}\right)$ acts as a scale factor on the size of the collapse.

The expected size of the bubble under the explosive regime is given by

$$
E_{t}\left(B_{t+1}\right)=q_{t} E_{t}\left(B_{t+1} \mid S_{t+1}=2\right)+\left(1-q_{t}\right) E_{t}\left(B_{t+1} \mid S_{t+1}=3\right) .
$$

Substituting (3) and rearranging yields

$$
E_{t}\left(B_{t+1} \mid S_{t+1}=2\right)=\left[(1+R) / q_{t}\right] B_{t}-1 / q_{t}\left[g\left(b_{t}\right) P_{t}\right] .
$$

Equation (7) shows that the expected bubble size in the explosive regime is a negative function of the probability $q_{t}$ of being in that regime. This implies that as the probability of being in the explosive regime decreases, investors demand a higher return to compensate for the risk of a possible collapse in the asset price. Thus, in the explosive regime, the gross return $(1+R) / q_{t}$ on the bubble exceeds the returns in the dormant and collapsing regimes.

In this paper, it is assumed that, conditional on the regime in period $t+1$, growth of the bubble is deterministic. Therefore, the bubble process evolves according to

$$
B_{t+1}=\left\{\begin{array}{lr}
(1+R) B_{t}, & \text { with probability } n_{t} \\
{\left[(1+R) / q_{t}\right] B_{t}-1 / q_{t} g\left(b_{t}\right) P_{t},} & \text { with probability }\left(1-n_{t}\right) q_{t} \\
g\left(b_{t}\right) P_{t} /\left(1-q_{t}\right), & \text { with probability }\left(1-n_{t}\right)\left(1-q_{t}\right) .
\end{array}\right.
$$

The probabilities of being in the dormant and explosive regimes $n_{t}$ and $q_{t}$ are negative functions of the bubble size. As the bubble size continues to grow in the dormant regime, the probability of entering the explosive regime increases, hence $n_{t}$ gets smaller. Likewise, as the bubble size continues to grow in the explosive regime the probability of the bubble collapsing increases, hence $q_{t}$ gets smaller.

To generalize the BK model, the probabilities $n_{t}$ and $q_{t}$ are specified to follow a first order Markov process. Specifically, the Markovian property requires that the current regime state indicator $S_{t+1}$ depends on its immediate past indicator $S_{t}$. As described above, in addition to the past state indicator, other conditioning driving variables $X_{t}$ are included in estimating the transition probabilities. Here, a probability matrix with a time-varying transition probability-generating function for each probability cell is specified. For the three-regime model, the time-varying transition probability function is written as

$$
P\left[S_{t+1}=i \mid S_{t}=j, X_{t}\right] \equiv P_{i j t}
$$

for $i, j=1,2,3$.

For the three-regimes, the probabilities can be written out as

$$
\begin{gathered}
P_{1 j t} \equiv P\left[S_{t+1}=1 \mid S_{t}=j, X_{t}\right]=n_{j t} \\
P_{2 j t} \equiv P\left[S_{t+1}=2 \mid S_{t}=j, X_{t}\right]=\left(1-n_{j t}\right) q_{j t} \\
P_{3 j t} \equiv P\left[S_{t+1}=3 \mid S_{t}=j, X_{t}\right]=\left(1-n_{j t}\right)\left(1-q_{j t}\right)
\end{gathered}
$$


with $\sum_{i=1}^{3} P_{i j t}=1$ as required.

The state variables in $X_{t}$ include the spread between fundamental returns and actual returns of the S\&P 500 index $S_{t}^{f, a}$, volume traded of the S\&P 500 index $V_{t}$, and the relative bubble size of the S\&P 500 index $b_{t}$. To constrain the probabilities between 0 and 1 , as well as ensuring that their sum equals 1 , simultaneously, the probit model specification of Ding (2012) is adopted. Specifically, we write

$$
\begin{gathered}
n_{j t}=\Phi\left(\alpha_{n, 0, j}+\alpha_{n, b, j} b_{t}+\alpha_{n, s, j} S_{t}^{f, a}\right) \\
q_{j t}=\Phi\left(\alpha_{q, 0, j}+\alpha_{q, b, j} b_{t}+\alpha_{q, v, j} V_{t}\right) \quad \text { for } j=1,2,3 .
\end{gathered}
$$

$\Phi()$ is the cumulative normal density function, $S_{t}^{f, a}$ represents the spread of the absolute value of the average 6-month actual returns and the absolute value of the average 6-month returns of the estimated fundamental values, and $V_{t}$ is the trade volume of the asset. The inclusion of the spread is to separate bubble returns from fundamental returns. This ensures that the higher (lower) the spread, the lower (higher) the probability of the bubble continuing to be in the dormant regime. To achieve this switch between regimes, the spread is introduced in the equation for the probability of being in a dormant regime. Doing so helps to identify the contribution of bubble returns in explaining average returns in period $t+1$.

Abnormal trade volume in the equation for the probability of being in an explosive regime serves as a signal to a possible bubble collapse. Based on the above, abnormal trade volume is added to the return equations in both explosive and collapsing regimes of the model, contrary to the BK model which only introduces abnormal trade volume in the explosive regime.

Trade volume $\left(V_{t}\right)$ is omitted from the probability of being in a dormant regime $\left(n_{j t}\right)$ because trade volumes do not experience large volatilities in the dormant regime of a bubble. Volatility in trade volume is negligible. With this identification structure, the spread and trade volume enter the probability equations separately.

The following signs are expected for the coefficient estimates of the probit models in equation (10).

$$
\begin{aligned}
& \begin{array}{l}
\alpha_{n, b, j}<0 \\
\alpha_{n, s, j}<0
\end{array} \\
& \begin{array}{l}
\alpha_{q, b, j}<0 \\
\alpha_{q, v, j}<0
\end{array} \\
& \left.\begin{array}{l}
\alpha_{n, b, j}>0 \\
\alpha_{n, s, j}>0
\end{array}\right\} \quad \text { for } j=1 \\
& \left.\begin{array}{l}
\alpha_{q, b, j}>0 \\
\alpha_{q, v, j}>0
\end{array}\right\} \quad \text { for } j \neq 1 \\
&
\end{aligned}
$$

Expressions (10a) and (10b) ensure that the probability of the bubble remaining dormant (explosive) decreases when the bubble size and spread (volume) increase. Similarly, (10c) and (10d) ensure that the probability of the bubble transitioning into a dormant (explosive) regime increases when the bubble size and spread (volume) increase.

\section{Asset Returns}

The bubble process above is applied to modelling the expected gross returns on an asset in each regime. The expected gross returns on an asset is given by

$$
E_{t}\left(r_{t+1}\right)=E_{t}\left[\left(P_{t+1}+D_{t+1}\right) / P_{t}\right]
$$

The expected gross returns in the dormant regime can be shown to be

$$
E_{t}\left(r_{t+1} \mid S_{t+1}=1\right)=1+R .
$$

This implies that in the dormant regime, the expected returns to an asset are equivalent to the fundamental returns. In the explosive regime, the expected returns to an asset is obtained as

$$
E_{t}\left(r_{t+1} \mid S_{t+1}=2\right)=(1+R)+\left(1 / q_{j t}\right)\left[\left(1-q_{j t}\right)(1+R) b_{t}-g\left(b_{t}\right)\right]
$$

Equation (13) indicates that investors take into account the probability of collapse once the bubble size continues to grow in the explosive regime. Given that they do not know when the bubble is likely to collapse they adjust their expectations of next period's gross returns by considering the probability of collapse. In the collapsing regime, the expected gross returns equation is given by

$$
E_{t}\left(r_{t+1} \mid S_{t+1}=3\right)=\left[(1+R)+g\left(b_{t}\right)\right] 1 /\left(1-q_{j t}\right)-(1+R) b_{t} .
$$


It is important to note that the expected bubble return is $1+R$ in the dormant regime, greater than $1+R$ in the explosive regime, and less than $I+R$ in the collapsing regime. This helps investors to correctly identify which regime they are in based on their realized returns.

The expected returns equations above are highly non-linear, so to estimate them the approach of van Norden and Schaller (1999) is followed. Here, a first-order Taylor expansion is derived, around some arbitrary $b_{0}$ and $v_{0}$. This yields

$$
r_{t+1}=\left\{\begin{array}{lr}
\beta_{1,0}+\mu_{t+1}^{1} & \text { if } b_{\mathrm{t}} \text { is dormant } \\
\beta_{2,0}+\beta_{2, b} b_{t}+\beta_{2, v} v_{t}+\mu_{t+1}^{2} & \text { if } \mathrm{b}_{\mathrm{t}} \text { is explosive } \\
\beta_{3,0}+\beta_{3, b} b_{t}+\beta_{3, v} v_{t}+\mu_{t+1}^{3} & \text { if } b_{\mathrm{t}} \text { is collapsing, }
\end{array}\right.
$$

where $b_{t}$ is the relative bubble size and $v_{t}$ is the abnormal share volume traded, and 1,2, and 3 represent the dormant, explosive and collapsing regimes, respectively. Returns in the dormant regime are not affected by the bubble size and abnormal volume since they are equivalent to the fundamental returns. Thus, they can be treated as a constant with some unexpected deviations. However, the returns in both explosive and collapsing regimes are functions of the relative bubble size and abnormal share volume as explained in previous sections. Once investors have been able to correctly predict returns in the next period, they will know what regime they are likely to face. Large positive returns will imply a higher probability of being in an explosive regime. Likewise, significantly low returns will imply a higher probability of being in a collapsing regime. Lastly, steady returns will denote a higher probability of being in a dormant regime. Hence, instead of identifying the bubble regime directly, identification is done by inferencing from the returns equations.

As argued by Brooks et al. (2005), results of the Taylor series expansion yield some testable implications with regard to the sign and magnitude of coefficient estimates from the three-regime model. These are required for the model to have explanatory power.

$$
\begin{gathered}
\beta_{2,0}>\beta_{1,0}>\beta_{3,0} \\
\beta_{2, b}>\beta_{3, b} \\
\beta_{3, b}<0 \\
\beta_{2, v}>0 \\
\beta_{3, v}<0
\end{gathered}
$$

Expression 15a is assumed to hold for the returns equations to yield the correct implications for the appropriate regime. On the other hand, $15 \mathrm{~b}$ to $15 \mathrm{e}$ are required to hold based on the implications of the Taylor series expansion. Expression (15a) implies that the returns in the explosive regime exceed those in the dormant and collapsing regimes, with the collapsing regime generating the least returns, in the absence of abnormal share volume and relative bubble size. Expression (15b) argues that as the bubble size increases, the expected returns in the explosive regime exceed the expected returns in the collapsing regime. Expression (15c) states that as the bubble collapses, the expected return on the asset should decrease. In (15d), expected returns in the explosive regime must increase if abnormal volume is observed, which signals an increase in the probability of a bubble collapse. Expression (15e) argues that an increase in abnormal share volume traded in the collapsing regime leads to negative returns on the asset.

It is important to note that the three-regime Markov switching model presented above is a generalization of the standard regime-switching BK model. By restricting the coefficients in the probabilities for the dormant and explosive regimes at time $t+1$ from varying with the regime existing at time $\mathrm{t}$, as well as setting the coefficient of abnormal trading volume in the returns equation for the collapsing regime to zero, the Markov regime-switching model reduces to the BK model. The BK model can be tested against the more general Markov regime-switching model under the null hypothesis the following set of parameter restrictions

$$
\begin{gathered}
\beta_{3, v}=0 \\
\alpha_{n, 0,1}=\alpha_{n, 0,2}=\alpha_{n, 0,3} \\
\alpha_{n, b, 1}=\alpha_{n, b, 2}=\alpha_{n, b, 3} \\
\alpha_{n, s, 1}=\alpha_{n, s, 2}=\alpha_{n, s, 3} \\
\alpha_{q, 0,1}=\alpha_{q, 0,2}=\alpha_{q, 0,3} \\
\alpha_{q, b, 1}=\alpha_{q, b, 2}=\alpha_{q, b, 3} \\
\alpha_{q, v, 1}=\alpha_{q, v, 2}=\alpha_{q, v, 3},
\end{gathered}
$$


against the alternative hypothesis that at least one of these restrictions is violated. A likelihood ratio test is used. Under the assumption of disturbance normality, the above Markov regime-switching model is estimated by conditional maximum likelihood. The conditional log-likelihood function for $r_{t+1}$ is given by;

$$
\ln L\left(r_{t+1} \mid r_{t}, \theta, X_{t}\right)=\sum_{t=1}^{T} \ln \left[\sum_{i=1}^{3} \sum_{j=1}^{3} f\left(r_{t+1} \mid S_{t+1}=i, S_{t}=j, r_{t}, \theta, X_{t}\right) P\left(S_{t+1}=i, S_{t}=j \mid r_{t}, \theta, X_{t}\right)\right]
$$

where $\theta$ is the vector of model parameters $(\alpha, \beta, \sigma), X_{t}$ is a vector of exogenous variables $\left(b_{t}, v_{t}, S_{t}^{f, a}\right)$ and $f()$ is the standard normal probability density function for each of the returns in (15). The log-likelihood function (16) represents a weighted average of the log-likelihood function in each regime with the weights being the transition probabilities. The latent transition probabilities are obtained using the Hamilton (1989) filter.

\section{Data}

Data for the study comprises 1,469 monthly observations on the S\&P 500 index from January 1888 to May 2010. Data on S\&P 500 index is available in monthly frequency as provided on Robert Shiller's website. It is worth noting here that, in cases where high frequency data such as weekly or daily data are available, they may not be ideal for the identification of an asset price bubble. A bubble phenomenon must be persistent, spanning a longer period. However, there are instances when asset prices undergo sharp price increases, which last for a few days or weeks. These events are characterized by a sudden 'craze' for a particular stock, especially during initial public offers (IPOs), with asset prices stabilizing after a few days or weeks. Such price increases represent fads and an attempt to identify bubbles using daily or weekly data will lead to a misclassification of such fads as bubbles. Therefore, the use of monthly data helps avoid this potential misclassification.

S\&P 500 price index and dividends are obtained from Robert Shiller's website. Data on abnormal volume is provided by Brooks, C. [see Brooks and Katsaris (2005) for details on how abnormal volume is calculated] who obtained it from the New York Stock Exchange (NYSE). Monthly dividend and price series are converted into real variables using monthly U.S consumer price index. All variables are seasonally adjusted. To calculate the spread, fundamental prices are first calculated using (17) and subsequently used to obtain fundamental returns. Actual returns are also calculated and the difference between the two returns are obtained using $S_{t}^{f, a}=\left|r_{t}^{a, 6}\right|-$ $\left|r_{t}^{f, 6}\right|$, where $r_{t}^{a, 6}$ is the average 6-month actual returns and $r_{t}^{f, 6}$ is the average 6-month returns of the estimated fundamental values. Figure 2 shows the bubble deviations of S\&P 500 price index from January 1888 to May 2010. As it turns out, the largest deviation was observed around the period of the Great Depression (1929-1933). For the period of the Great Recession (2007-2009), a negative deviation of the bubble size is observed. It is observed from Figure 2 that large deviations are associated with low asset prices. In general, the bubble deviation and the S\&P 500 price index exhibit similar patterns over time.

To estimate the three-regime model, fundamental values of the S\&P 500 index are first determined. Under the assumption that the dividend process follows a random walk with drift, it can be shown that

$$
P_{t}^{f}=\rho D_{t},
$$

where $\rho$ is the sample mean of the price-dividend ratio. Having obtained the fundamental values, the relative bubble size $b_{t}$ is then calculated as $b_{t}=1-\rho\left(D_{t} / P_{t}\right)$.

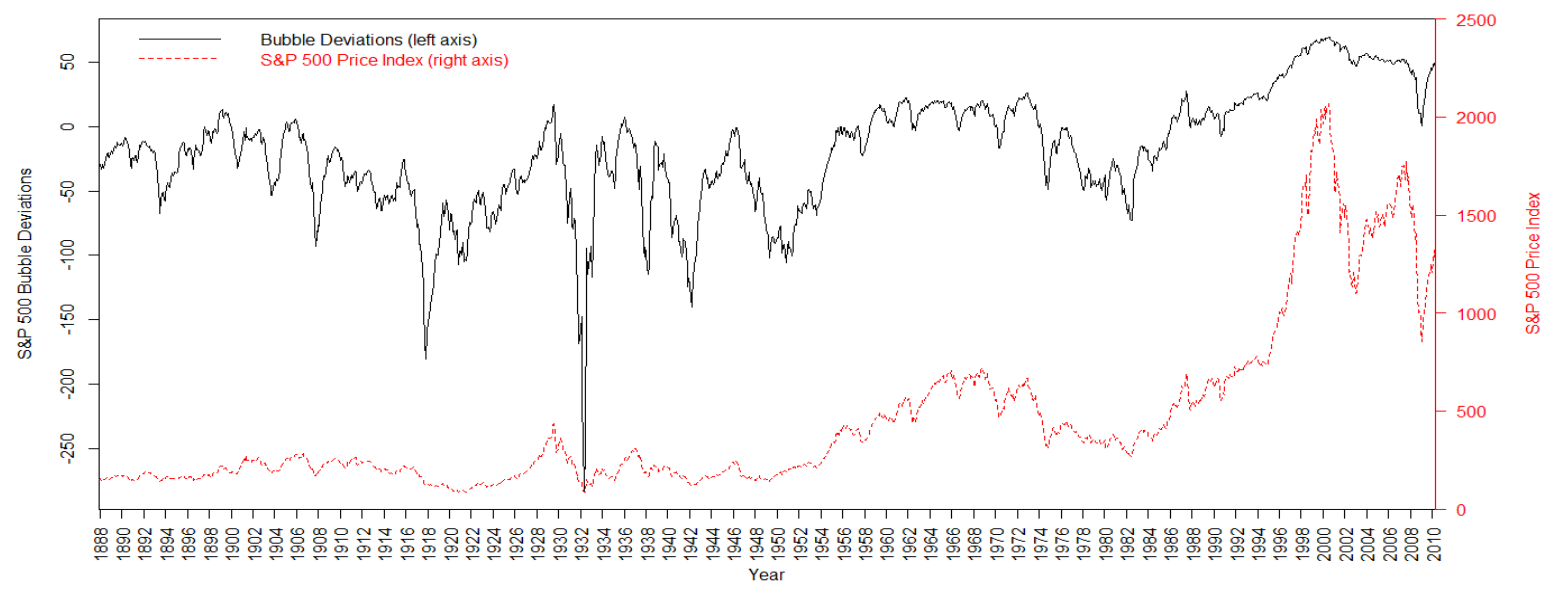

Figure 2. Bubble deviations and S\&P 500 price index from January 1888 to May 2010 


\section{Results}

In this section, we present results from the three-regime Markov switching model and the BK model. A likelihood ratio (LR) test is conducted to examine the appropriateness of the BK regime-switching model against the more general Markov regime-switching model. The restrictions as noted above are the exclusion of abnormal share trading volume in the returns equation for the collapsing regime as well as the homogeneity of transition probability coefficients across the bubble regimes. Results are presented at the bottom of Table 1 . The LR test strongly rejects the BK model against the Markov regime-switching model at a $1 \%$ level of significance. The coefficient estimates in Table 1 indicate that the rejection is due to both the Markov regime-switching structure and the inclusion of trade volume in both explosive and collapsing regimes.

Despite the rejection of the BK model against the Markov regime-switching, there are some points of agreement in the results from both models. The two models largely agree on the contribution of bubbles to the returns on an asset as shown by the beta coefficients in Table 1. The coefficients exhibit the same signs but different magnitudes in both models. One notable difference is the inclusion of trade volume in the returns equation corresponding to the Markov regime-switching model which turns out to be highly significant.

The major difference between the two models lies in the time-varying transition probabilities. Whereas the Markov regime-switching model finds a negative and significant effect, as indicated by $\alpha_{n, b, 1}$, from relative bubble size on the probability of the S\&P 500 index remaining in the dormant regime, this is not the case in the BK model. In the original BK model however, this coefficient was significant. The likely reason for these results could be the difference in sample periods used. Results from the original BK model are based on a sample from 1888 to 2003 whereas this study uses a sample from 1888 to 2010. This represents the inclusion of seven more years of monthly data. Although this may not be a wide gap, between 2003 and 2010 the Great Recession occurred, and the sheer magnitude of this recession could be a significant contributor to this difference in results from the two models. Another point of departure between the Markov regime-switching model and BK model is observed in the transition probabilities for the explosive regime.

Table 1. Results of the three-regime rational speculative bubble model

\begin{tabular}{|c|c|c|c|c|}
\hline \multirow[b]{2}{*}{ Coefficient } & \multicolumn{2}{|c|}{ Markov Regime-Switching Model } & \multicolumn{2}{|c|}{ BK Regime Switching Model } \\
\hline & Value & Standard Error & Value & Standard Error \\
\hline$\beta_{1,0}$ & $0.0052 *$ & 0.0013 & $0.0086^{*}$ & 0.0011 \\
\hline$\beta_{2,0}$ & $0.0094 *$ & 0.0021 & $0.0101 *$ & 0.0049 \\
\hline$\beta_{3,0}$ & $-0.0224 *$ & 0.0098 & $-0.0298^{*}$ & 0.0127 \\
\hline$\beta_{2, b}$ & $1.6892 *$ & 0.5001 & $3.6805^{*}$ & 1.5160 \\
\hline$\beta_{3, b}$ & $-4.1854^{*}$ & 1.2159 & $-1.5555^{* *}$ & 0.8069 \\
\hline$\beta_{2, v}$ & $0.1084^{*}$ & 0.0045 & $0.1504 *$ & 0.0109 \\
\hline$\beta_{3, v}$ & $-0.0744 *$ & 0.0284 & - & - \\
\hline$\alpha_{n, 0,1}$ & $2.9567 *$ & 0.6106 & $1.7961^{*}$ & 0.3170 \\
\hline$\alpha_{n, b, 1}$ & $-137.8270^{* *}$ & 75.9454 & 43.5117 & 38.3423 \\
\hline$\alpha_{n, s, 1}$ & $-111.7375^{*}$ & 30.6523 & $-81.7089 *$ & 22.3635 \\
\hline$\alpha_{n, 0,2}$ & 0.1842 & 0.6897 & - & - \\
\hline$\alpha_{n, b, 2}$ & -63.3835 & 84.4041 & - & - \\
\hline$\alpha_{n, s, 2}$ & -27.6364 & 24.4293 & - & - \\
\hline$\alpha_{n, 0,3}$ & $-1.7177 *$ & 0.3924 & - & - \\
\hline$\alpha_{n, b, 3}$ & 55.5329 & 83.0472 & - & - \\
\hline$\alpha_{n, s, 3}$ & -62.5901 & 46.1321 & - & - \\
\hline$\alpha_{q, 0,1}$ & 7.9098 & 50.9511 & $1.1677^{*}$ & 0.4854 \\
\hline$\alpha_{q, b, 1}$ & 6.9507 & 522.0242 & 102.0700 & 83.1074 \\
\hline$\alpha_{q, v, 1}$ & -7.9515 & 55.9233 & $-1.9386^{* *}$ & 1.1610 \\
\hline$\alpha_{q, 0,2}$ & 0.2358 & 0.3196 & - & - \\
\hline$\alpha_{q, b, 2}$ & 7.8665 & 31.7039 & - & - \\
\hline$\alpha_{q, v, 2}$ & 1.0922 & 1.0387 & - & - \\
\hline$\alpha_{q, 0,3}$ & $-2.1642 *$ & 0.3346 & - & - \\
\hline$\alpha_{q, b, 3}$ & $116.9485^{*}$ & 55.7337 & - & - \\
\hline$\alpha_{q, v, 3}$ & -0.7385 & 1.0050 & - & - \\
\hline$\sigma_{1}$ & $0.0008 *$ & 0.0001 & $0.0008^{*}$ & 0.0001 \\
\hline$\sigma_{2}$ & $0.0010^{*}$ & 0.0001 & $0.0011^{*}$ & 0.0001 \\
\hline$\sigma_{3}$ & $0.0035^{*}$ & 0.0010 & $0.0047 *$ & 0.0007 \\
\hline
\end{tabular}




\begin{tabular}{lcc}
\hline Likelihood & 2801.9178 & 2685.2671 \\
LR Test & & \\
Test statistic & 233.3014 & \\
P-value & 0.0000 & \\
\hline
\end{tabular}

Note. All variables are in logarithms in both models. Both models are estimated using S\&P 500 data from January 1888 to May 2010 . The BK model is estimated with restrictions noted in Brooks and Katsaris (2005). *, ** represent 5\% and 10\% level of significance, respectively.

Here, results from the Markov regime-switching model show that once the bubble enters the explosive regime, the probability of remaining in the explosive regime does not depend on the relative bubble size. Once the explosive regime occurs the bubble sustains itself and grows with a fixed probability. Evidence in support of this is the prolonged period of growth in the S\&P 500 index from 1990 to 2000 (during the technology bubble) and again from 2002 to 2008 (during the housing bubble). The final distinction between the Markov regime-switching model and the BK model lies in the behavior of bubbles in the collapsing regime. Once in a collapsing regime, as the bubble size begins to grow the probability of transitioning into an explosive regime increases. This phenomenon of reverting from a collapsing regime immediately to an explosive regime was observed in the S\&P 500 index as recently as 2003 following the collapse of the technology bubble and in 2009 after the crash as observed in Figure 1. The remainder of this section provides a detailed discussion of the results.

We first consider the coefficients in the returns equation (15). From Table 1, the estimate of the intercept in the dormant regime $\beta_{1,0}$ implies an average return of $0.52 \%$ per month $(6.42 \%$ effective annual rate) whereas it is $0.86 \%$ per month in the BK model (10.82\% effective annual rate). This magnitude is reasonable in the context of a bubble developing before transitioning into an explosive state. The initial realization of the presence of a bubble causes more investors to enter the market, bidding the price of the asset upwards. This leads to increased returns in the dormant phase of the bubble. When the explosive regime is reached, the average returns $\beta_{2,0}$ on the asset independent of the bubble size and abnormal volume stands at $0.94 \%$ per month $(11.88 \%$ effective annual rate). This is roughly twice what is realized in the dormant regime. In the BK model, average returns of $1.01 \%$ per month (12.68\% effective annual rate) is realized. During this period, exponential growth in asset prices ensures that returns far exceed what is obtained in the dormant regime. More investors are attracted to the market to buy the asset and resell to another investor at a higher price in future. However, as several investors soon offer their asset holdings for sale, excess supply causes price to tumble. This ushers in the collapsing regime, where the return on the asset $\beta_{3,0}$ is $-2.24 \%$ per month $(23.80 \%$ effective annual rate). The average returns are $-2.98 \%$ per month (30.44\% effective annual rate) in the BK model. At this rate, the gains in the previous regime are soon eroded. It is observed that the BK model consistently overstates the average returns on the asset. This could result from the model's lack of consideration for what regime was experienced in the previous period.

Turning now to the slope coefficients, bubbles in the two regimes have the expected signs and are significant at $5 \%$. It is observed that the size of the bubble contributes about $1.69 \%$ returns to the asset in the explosive regime $\beta_{2, b}$ for every $1 \%$ increase in the bubble size and $-4.19 \%$ in the collapsing regime $\beta_{3, b}$ for every $1 \%$ decrease in the bubble size. The positive sign of the bubble in the explosive regime confirms the notion that as the bubble increases in size investors demand higher returns to compensate them for the risk of a potential bubble collapse.

It is also observed from the table that abnormal share volume has a significant impact on asset returns in both explosive and collapsing regimes. In the explosive regime, the average return on the asset $\beta_{2, V}$ increases by $0.11 \%$ per month for a $1 \%$ increase in trading volume. This increase agrees with the prediction of the rational speculative bubble model since investors make positive gains from trading assets in the explosive regime. In the collapsing regime, average returns on the S\&P 500 index $\beta_{3, V}$ decrease by $0.07 \%$ per month for every $1 \%$ decrease in trading volume. Recall that the abnormal share volume traded was excluded from the returns equation in the collapsing regime in the BK model. The observed negative and significant impact of abnormal share volume traded on asset returns justifies its inclusion in the returns equation for the collapsing regime in the more general Markov regime-switching model. Inclusion of abnormal share volume traded in the collapsing regime is also consistent with the historical losses observed in the S\&P 500 during a bubble collapse. On 'Black Monday' October 19, 1987, the S\&P 500 lost about $20.5 \%$ of its value which was the largest loss ever experienced by any index in a day. Consequently, large average trading volume was experienced, standing at $\$ 277,026,455.45$ in October 1987 compared to \$177,318,727.14 in September 1987. Between January 20, 2009 and March 9, 2009 during the financial crisis, the S\&P 500 index lost about 15.90\% of its value. These market collapses are all characterized by abnormal share volumes traded during these periods. The S\&P 500 saw average share volume traded increasing from \$1.3trillion to \$1.8trillion between January and March 2009 (Based 
on data from the NYSE obtained by Chris Brooks).

Results of the time-varying transition probabilities (TVTPs) provide another point of departure of the Markov regime-switching model from the BK model. Whereas the Markov switching model estimates a different probability for transitioning between any two regimes or even the same regime from period $t$ to $t+1$, the BK model implicitly assumes that the transition probabilities depend only on the exogenous variables. This implies that $P_{11 t}=P_{12 t}=P_{13 t} \equiv n_{j t}$ in the dormant regime, $P_{21 t}=P_{22 t}=P_{23 t} \equiv q_{j t}$ in the explosive regime, and $P_{31 t}=P_{32 t}=P_{33 t} \equiv 1-q_{j t}$ in the collapsing regime. However, in the Markov regime-switching model considered here, nine separate probabilities are obtained, regarding every possible transition between the three regimes.

We first consider the parameters in $n_{j t}$, which determine the probability of being in the dormant regime. From Table 1, the intercept $\alpha_{n, 0,1}$ suggests that there is a $99.84 \%$ [obtained by $\Phi\left(\alpha_{n, 0,1}\right)$ ] probability of the bubble remaining in a dormant regime when the bubble size and spread are zero. The negative and significant coefficient on the bubble size $\alpha_{n, b, 1}$ indicates that as the bubble size grows, the probability of being in the dormant (explosive) regime in period $t+1$ decreases (increases). Similarly, as the spread increases, the probability of remaining in the dormant regime decreases, as evident from the coefficient of the spread $\alpha_{n, s, 1}$. This is a reasonable outcome as an increase in the spread can only happen when the bubble size is increasing, thus, reducing the likelihood of being in the dormant regime. The probability of transitioning from an explosive regime to a dormant regime did not yield any significant coefficients. This may suggest that the possibility of a reverse transition from an explosive to a dormant regime is limited, although this phenomenon was observed between 1990 and 1994. The intercept $\alpha_{n, 0,3}$ in the equation for the probability of transitioning from a collapsing regime to a dormant regime is negative and significant. It signifies a $4.29 \%$ [obtained from 1$\left.\Phi\left(\alpha_{n, 0,3}\right)\right]$ probability of the bubble transitioning from the collapsing regime to the dormant regime.

We next consider the parameters in $q_{j t}$, which determine the probability of an explosive regime occurring. We find that $\alpha_{q, b, 2}$ and $\alpha_{q, v, 2}$ are not significantly different from zero indicating that the bubble size and trading volume do not significantly impact the probability of an explosive regime continuing to the next period. The fact that we find $\alpha_{n, b, 2}=\alpha_{n, s, 2}=\alpha_{q, b, 2}=\alpha_{q, v, 2}=0$ implies that once the bubble enters the explosive regime, the bubble dynamics are independent of the state variables. When the bubble is in the explosive regime, the probability that the bubble remains in the explosive regime or transitions to the collapsing or dormant regimes is constant. The coefficient of the bubble size in the probability of switching from a collapsing regime to an explosive regime $\alpha_{q, b, 3}$ is positive and significant. This implies that as the bubble size grows in the collapsing regime, the probability of an explosive regime occurring the next period increases. This phenomenon was observed in the S\&P 500 index between 2002 and 2010 when bubble collapses were immediately followed by the emergence of an explosive bubble.

Lastly, as the rational speculative bubble model predicts, the standard deviations of residuals from the three-regime Markov switching model yield the desired results. The standard deviation of the residuals in the collapsing regime exceed that in the explosive regime which in turn exceeds the standard deviation in the dormant regime. From Table 1, the standard deviations for the residuals in the dormant, explosive and collapsing regimes are $0.0008 \%, 0.0010 \%$, and $0.0035 \%$, respectively. The high standard deviation in the collapsing regime is consistent with experience because collapsing bubbles are associated with highly volatile negative returns.

Figures 3, 4, and 5 plot the unconditional probabilities of being in a dormant regime, an explosive regime, and a collapsing regime, respectively, together with the S\&P 500 price index from January 1888 to May 2010. In general, the three-regime Markov switching process performs well at identifying periods of asset price bubbles.

Figure 3 shows that in periods when the S\&P was relatively stable, the probability of observing a dormant bubble was high and remained so for an extended period from1888 to about 1930. Except for some periods of brief collapse in the S\&P 500 such as $1901,1904,1908,1922,1929$, the index was relatively stable up until the Great Depression. The year 1901 witnessed a stock market panic, lasting 3 years as well as the year-long panic of 1907. Another significant observation here is that as the bubble size increases over time, the probability of being in a dormant regime decreases, signaling the onset of an explosive regime.

Figure 4 represents the probability of being in an explosive regime as the bubble size grows over time. It is observed that these probabilities remained low for an extended period from 1888 to 1898 and again from 1907 until the Great Depression. The observed behavior is characteristic of the dormant nature of the S\&P 500 before the Great Depression as well as the collapse during the Great Depression. As expected, the probability of an explosive bubble is close to zero for the Great Depression period, as there was no possibility for an explosive bubble to occur during the period. Although the model correctly identifies this, it fails however, to identify the 
rising prices before the depression. It captures well the price rally from 1956 to 1966 in the S\&P 500, following in that direction up to the 1990s when a price rally was more pronounced because of the internet bubble. With the bubble collapsing in 2000, a lower probability of an explosive bubble is observed until 2002. A price rally re-emerged during this period until its eventual collapse in 2007 during the Great Recession, evidenced by a low probability of being in an explosive regime.

Lastly, Figure 5 which reports the probabilities of being in a collapsing regime shows a large increase in the probability of a bubble collapsing during the Great Depression from 1929 to 1933 . The mild Kennedy crash of 1962, also called the 'flash crash' is correctly identified by the model, as the probability of being in a collapsing regime increases sharply during the period. The stock market crash of 1970 and 1974 are also correctly identified with very high probabilities of a collapse. The most recent market crashes are seen in 1987, 1989, 2000 and 2007 on 'Black Monday,' 'Friday the 13th,' the collapse of the technology bubble, and the Great Recession, respectively.

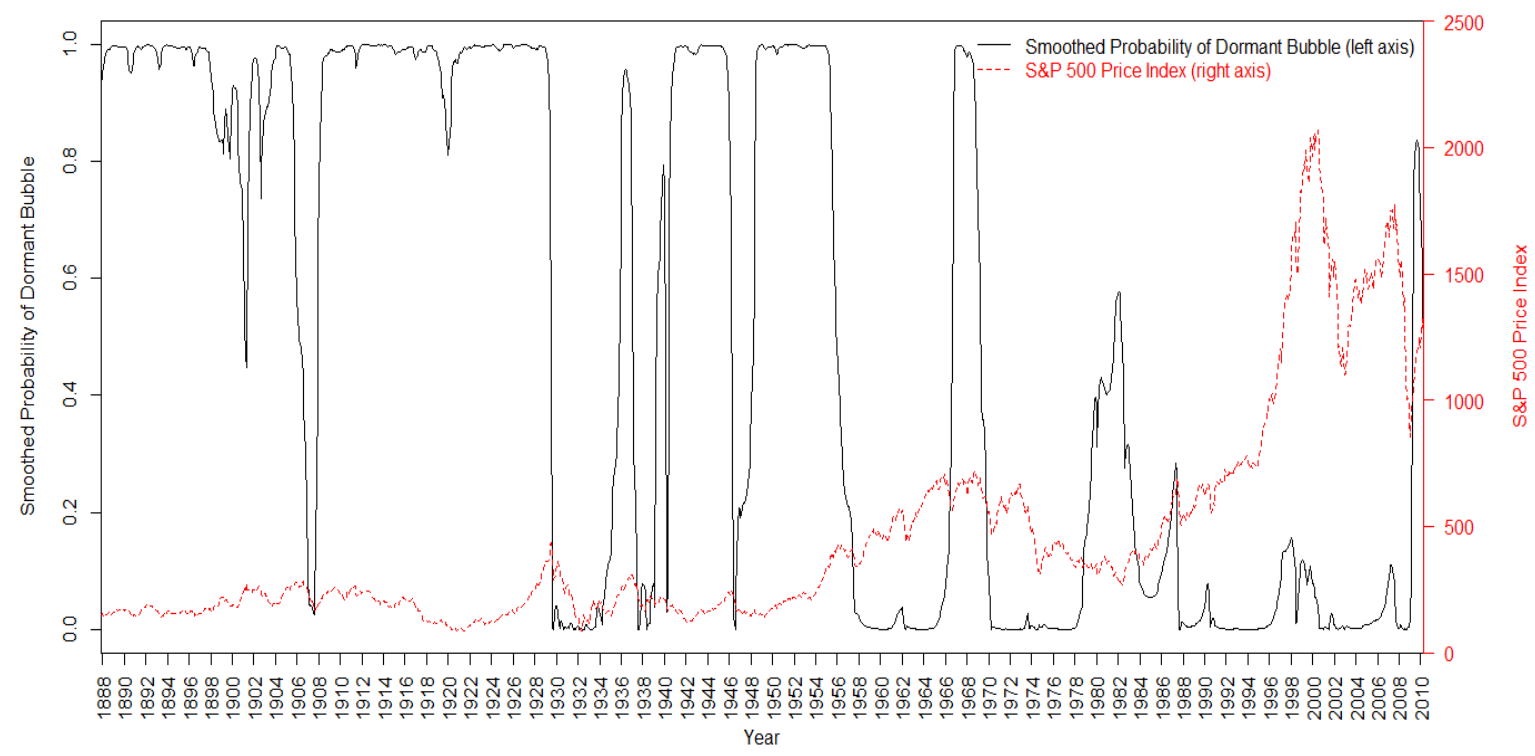

Figure 3. Smoothed probabilities of a dormant bubble

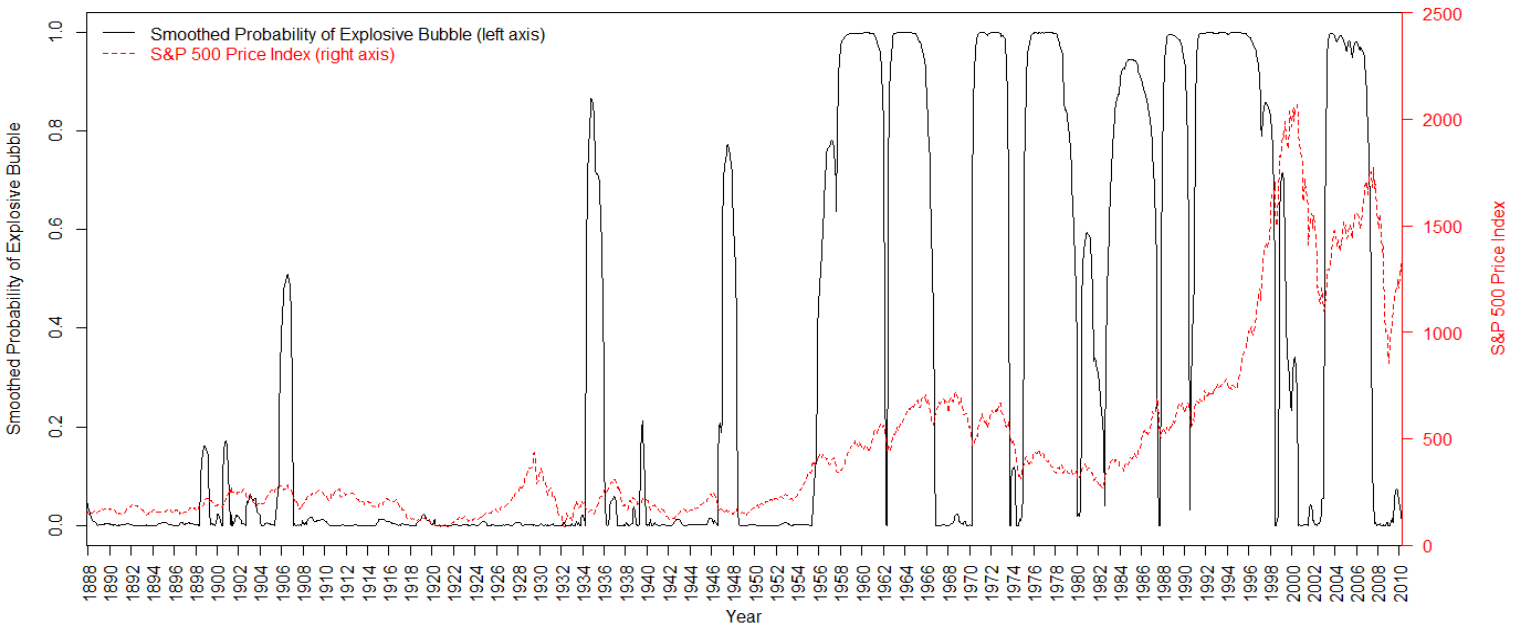

Figure 4. Smoothed probabilities of an explosive bubble

\section{Robustness Analysis}

In this section, two main robustness analyses are conducted. First, the sample period is split into two parts; from January 1888 to December 1950 (756 observations) and from January 1951 to May 2010 (713 observations). 
This split is based on the fact that after World War II, stock markets were relatively stable with the evolution of Keynesian economic policies until the oil crisis in the 1970s. The choice of 1950 is to allow sufficient time after World War II so that the adverse economic effects of the war would have subsided, giving room for economic policies to function.

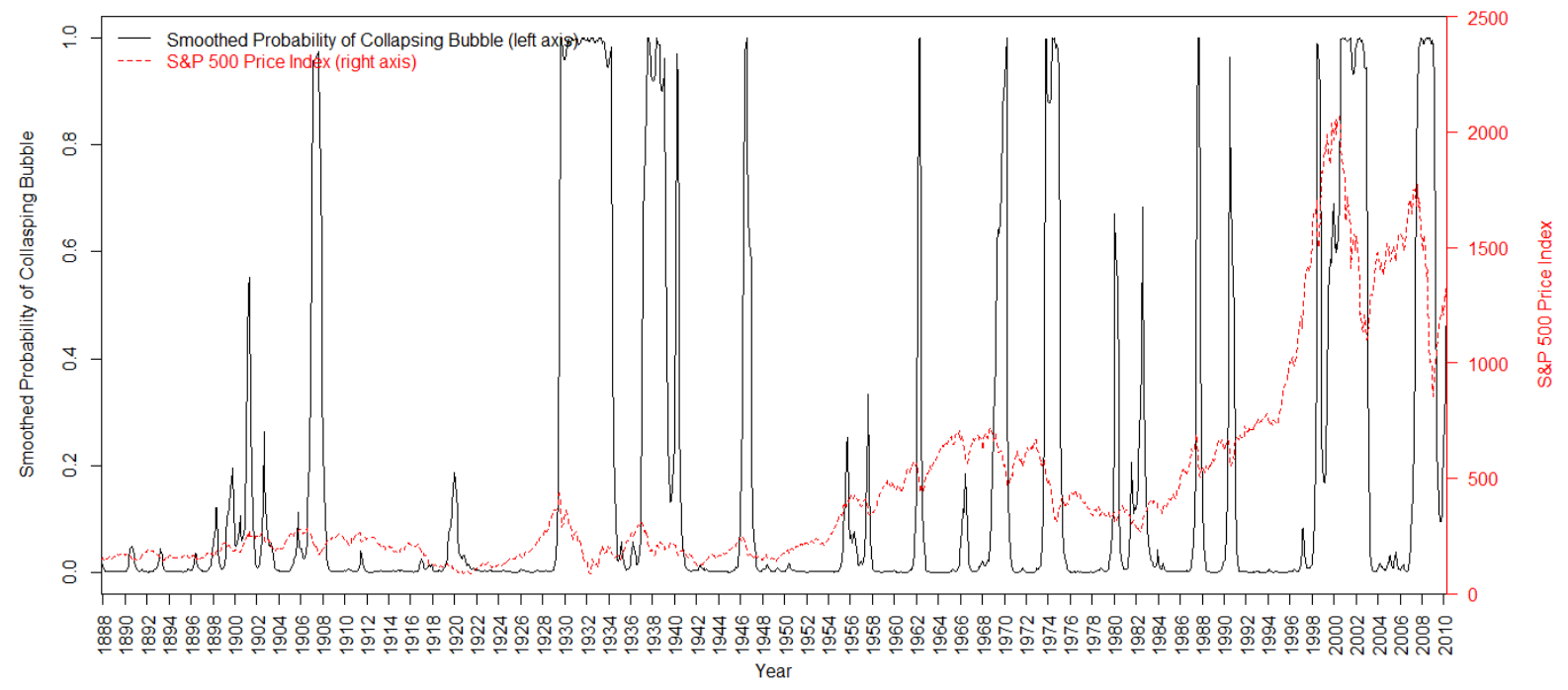

Figure 5. Smoothed probabilities of a collapsing bubble

Second, 1-period lagged values of the spread between the actual returns and fundamental returns on the S\&P 500 index are used in estimating the model for the full sample. This change is based on the idea that the returns on an asset in the immediate past period will provide more information about the existing bubble regime in period $t$ than would returns for the past six months. Thus, 1-period lagged values of the spread are expected to provide sharper identification for asset price bubbles in period $t$.

Results based on data splitting are shown in Figures 6 and 7, showing a plot of the smoothed probabilities of being in a dormant, explosive, and collapsing bubble regime. It is observed that results based on the full sample are upheld by those based on the split data. Similarly, results based on 1-period lagged spread values, reported in Figure 8 are consistent with those obtained in Figures 1, 2, and 3.

Based on the results obtained, it can be concluded that the Markov regime-switching model works well in identifying asset price bubbles. Results are not dependent on the sample period or how the spread is measured.

\section{Conclusion}

A three-regime Markov switching model was developed to identify asset price bubbles in the S\&P 500 composite price index, using data from January 1888 to May 2010. Regimes were classified into dormant, explosive, and collapsing, with each characterizing the observed behavior of the S\&P 500 index over the period. With the observed relationship between asset price bubbles and asset returns, return equations were subsequently formulated to provide a more flexible approach to identifying bubbles. Time-varying transition probabilities were formulated to account for every possible type of transition among the regimes. With a three-regime model, nine time-varying transition probabilities were formulated using a probit model to restrict the size between 0 and 1.

Two representations of the results from the three-regime Markov switching rational speculative bubble model are provided. First, coefficient estimates are provided in Table 1 and compared with those obtained in the BK model. Second, probability plots based on the coefficient estimates are presented for each regime in Figures 3, 4, and 5. Intercept terms show that on average returns on the S\&P 500 index increase by about $0.52 \%$ per month in the dormant bubble regime, $0.94 \%$ in the explosive regime and decrease by $2.22 \%$ in the collapsing regime. The bubble size as well as abnormal share volume traded impact asset returns significantly in the explosive and collapsing regimes. On the time-varying transition probabilities, it is observed that the spread between actual returns and fundamental returns negatively affects the probability of remaining in a dormant bubble regime. On the probability plots, it is observed from Figures 3, 4, and 5 that the three-regime rational speculative bubble model is able to identify most of the historical bubble phenomena, such as 'The Great Depression,' 'Black 
Monday,' 'Friday the 13th,' the 'Kennedy slide (flash crash),' and the 'Internet Bubble.' The above results show that the rational speculative bubble model accurately identifies multiple historical bubbles. It performs better than the BK model which fails to identify some historical bubble crashes such as observed in 1962 (the Kennedy slide), 1987 (Black Monday), 1989 (Friday the 13th), and 2000 (the technology bubble). The Markov regime switching model therefore has more explanatory power relative to the standard regime switching model. The likelihood ratio test also provides evidence that the Markov regime-switching model is a better specification for analyzing bubbles in the S\&P 500 index.
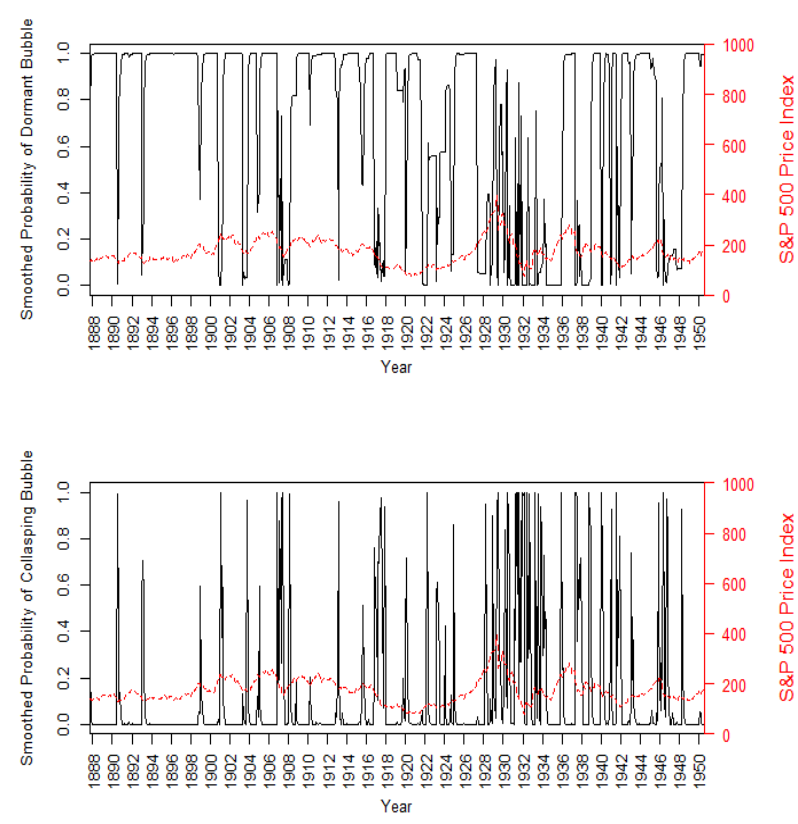

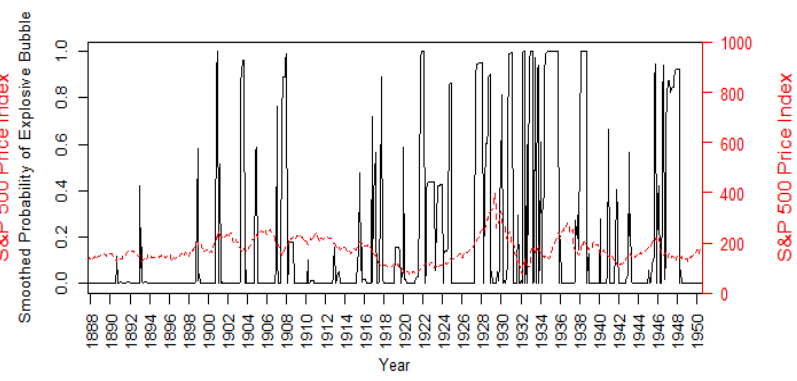

Year

Figure 6. Smoothed probabilities of a dormant, explosive, and collapsing bubble, 1888 to 1950
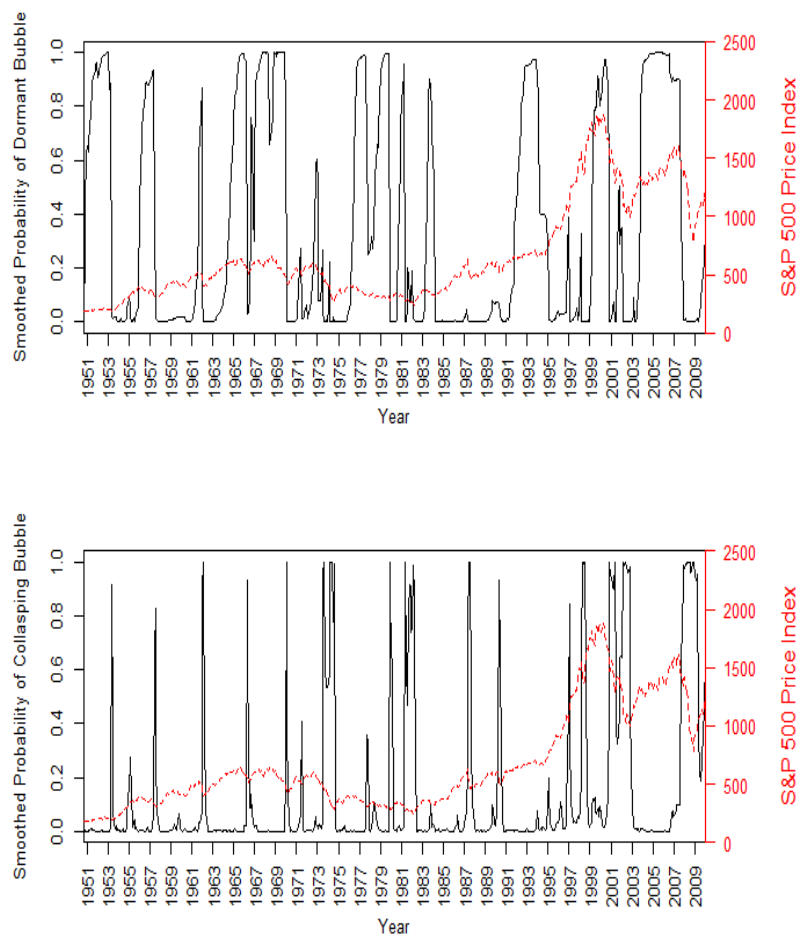

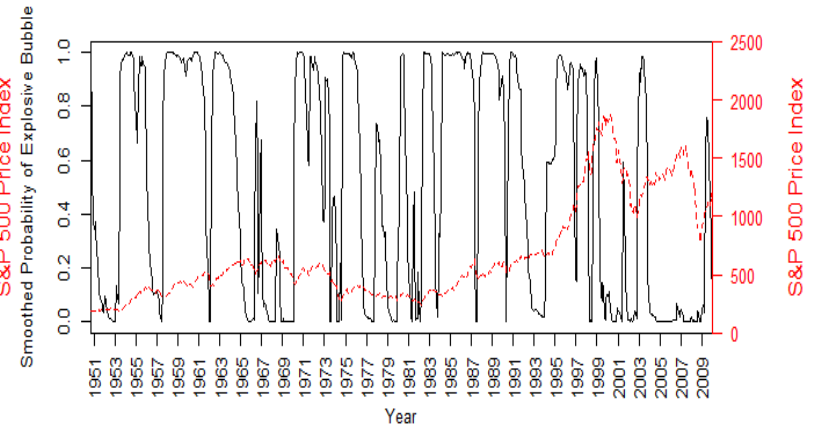

$\times$
0
0
0
0
0
0
0
0
0
0
0
0
0
0

Figure 7. Smoothed probabilities of a dormant, explosive, and collapsing bubble, 1951 to 2010 

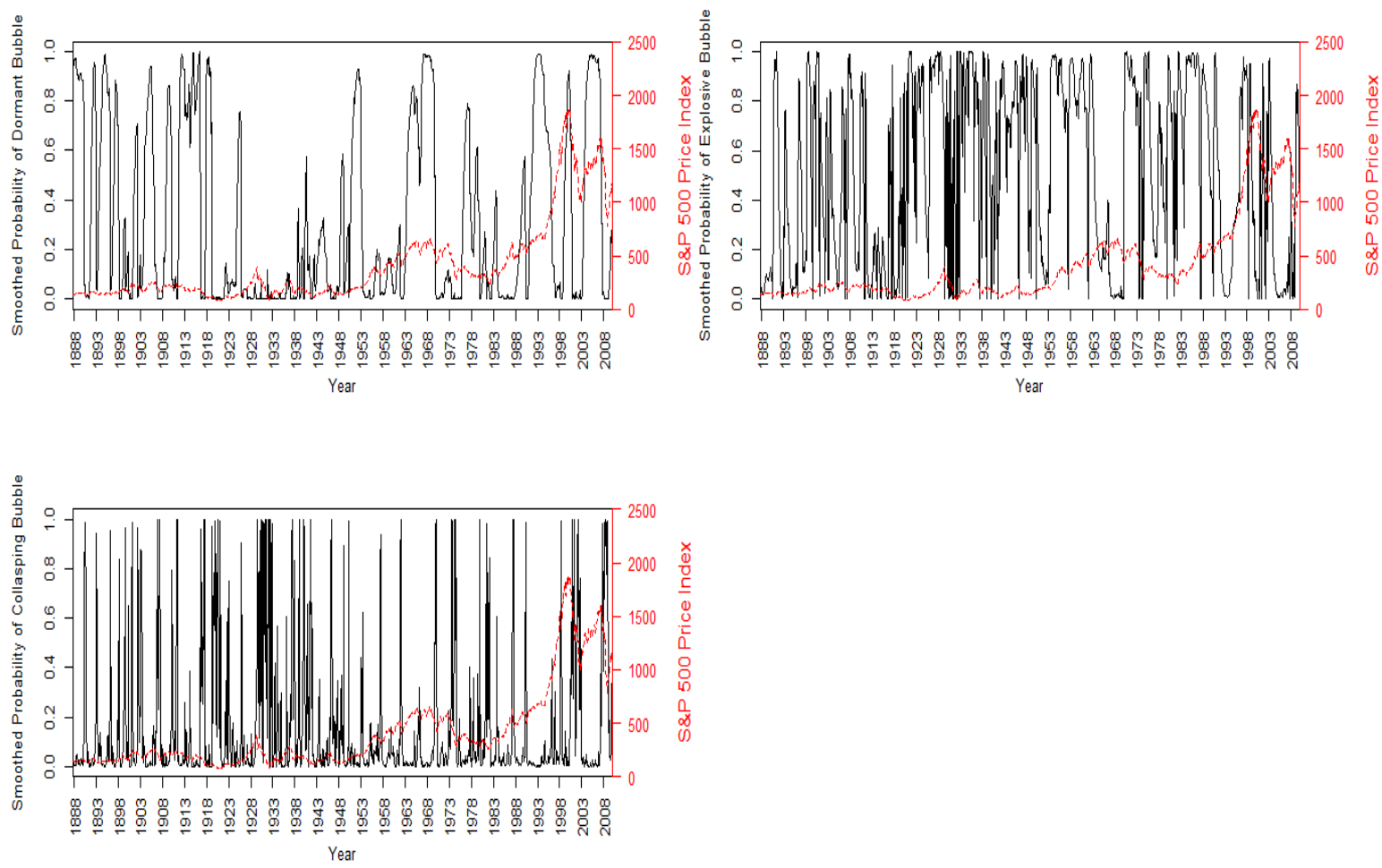

Figure 8. Smoothed probabilities of a dormant, explosive, and collapsing bubble, 1888 to 2010

\section{References}

Anderson, K., Brooks, C., \& Katsaris, A. (2010). Speculative Bubbles in the S\&P 500: Was the Tech Bubble Confined to the Tech Sector? Journal of Empirical Finance, 17, 345-361. https://doi.org/10.1016/j.jempfin.2009.12.004

Balke, N. S., \& Wohar, M. E. (2009). Market Fundamentals versus Rational Bubbles in Stock Prices: A Bayesian Perspective. Journal of Applied Economics, 24(1), 35-75. http://dx.doi.org/10.1002/jae.1025

Brooks, C., \& Katsaris, A. (2005). A Three-Regime Model of Speculative Behaviour: Modelling the Evolution of the S\&P 500Composite Index. The Economic Journal, 115(505), 767-797. http://dx.doi.org/10.1111/j.1468-0297.2005.01019.x

Diba, B. T., \& Grossman, H. I. (1987). On the Inception of Rational Bubbles. The Quarterly Journal of Economics, 102(3), 697-700. https://doi.org/10.2307/1884225

Diba, B. T., \& Grossman, H. I. (1988). The Theory of Rational Bubbles in Stock Prices. The Economic Journal, 98(392), 746-754. https://doi.org/10.2307/2233912

Ding, Z. (2012). An Implementation of Markov Regime Switching Model with Time Varying Transition Probabilities in MATLAB. http://dx.doi.org/10.2139/ssrn.2083332

Evans, G. W. (1991). Pitfalls in Testing for Explosive Bubbles in Asset Prices. The American Economic Review, 81(4), 922-930.

Fulop, A., \& Yu, J. (2017). Bayesian analysis of bubbles in asset prices. Working Paper.

Hamilton, J. D. (1989). A New Approach to The Economic Analysis of Nonstationary Time Series and the Business Cycle. Econometrica, 5(2), 357-384. https://doi.org/10.2307/1912559

http://www.sniper.at/stock-market-crash-of-1987.htm

https://en.wikipedia.org/wiki/United_States_bear_market_of_2007\%E2\%80\%9309

Phillips, P. C. B., Shi, S., \& Yu, J. (2015a). Testing for Multiple Bubbles: Historical Episodes of Exuberance and Collapse in the S\&P 500. International Economic Review, 56(4), 1043-1078. http://dx.doi.org/10.1111/iere.12132

Phillips, P. C. B., Shi, S., \& Yu, J. (2015b). Testing for Multiple Bubbles: Limit Theory of Real-Time Detectors. 
International Economic Review, 56(4), 1079-1134. http://dx.doi.org/10.1111/iere.12131

Phillips, P. C. B., Wu, Y., \& Yu, J. (2011). Explosive Behavior in the 1990s NASDAQ: When did Exuberance Escalate Asset Values? International Economic Review, 52(1), 201-226. http://dx.doi.org/10.1111/j.1468-2354.2010.00625.x

Schaller, H., \& Van Norden S. (1999). Speculative Behaviour, Regime-Switching, and Stock Market Crashes. In P. Rothman (Ed.), Nonlinear Time Series Analysis of Economic and Financial Data (pp. 321-56). Dordrecht Kluwer.

Schaller, H., \& Van Norden, S. (1993). The Predictability of Stock Market Regime: Evidence from the Toronto Stock Exchange. Review of Economics and Statistics, 75, 505-510. https://doi.org/10.2307/2109465

Schaller, H., \& Van Norden, S. (2002). Fads or Bubbles? Empirical Economics, 27, 335-362. https://doi.org/10.1007/s001810100116

Shi, S. P., \& Song, Y. (2014). Identifying Speculative Bubbles using an Infinite Hidden Markov Model. Journal of Financial Econometrics, 14(1), 159-184. https://doi.org/10.1093/jjfinec/nbu025

Shiller, R. J. (n. d.). U.S. Stock Markets 1871 - Present and CAPE Ratio. Retrieved from http://www.econ.yale.edu/ shiller/data.htm

The National Bureau of Economic Research (NBER). US Business Cycle Expansions and Contractions. Retrieved from http://www.nber.org/cycles.html

Van Norden, S., \& Vigfusson, R. (1998). Avoiding the Pitfalls: Can Regime-switching Tests Reliably Detect Bubbles? Studies in Non-Linear Dynamics and Econometrics, 3(1), 1-22. https://doi.org/10.2202/1558-3708.1038

\section{Copyrights}

Copyright for this article is retained by the author(s), with first publication rights granted to the journal.

This is an open-access article distributed under the terms and conditions of the Creative Commons Attribution license (http://creativecommons.org/licenses/by/4.0/). 